\title{
Caracterización de los módulos de los distritos de riego y presencia de organizaciones locales
}

\section{Characterization of Irrigation District Water User Associations and Presence of Local Organizations}

\author{
Jacinta Palerm Viqueira* (iD) https://orcid.org/0000-0002-5499-9791
}

\begin{abstract}
Resumen
Los módulos de los distritos de riego a pesar de contar con autogobierno tienen rasgos que difieren de aquellos de organizaciones de riego tradicionales con autogobierno. El primer objetivo del presente trabajo es caracterizar esos rasgos distintivos. El segundo objetivo es explorar la presencia de organizaciones locales informales en los módulos. Se emplean dos métodos: el comparativo (entre tipos de organizaciones y entre módulos) y el método etnográfico (en los módulos de riego). Los resultados son la caracterización de los rasgos distintivos y la documentación sobre la presencia de organizaciones locales en los módulos. Los estudios de caso de módulos, en relación con el universo de distritos de riego son limitados. No obstante, el abordaje es novedoso. Pocos estudios contrastan dichas organizaciones y hay pocas etnografías de lo local en los módulos. Se muestra que hay diferencias sustantivas entre esas organizaciones y que, no obstante la rigidez y la centralización del diseño organizativo de los módulos, hay en éstos efervescencia organizativa local. Palabras clave: México; módulos; distritos de riego; administración burocrática; organizaciones locales.
\end{abstract}

\begin{abstract}
The irrigation districts water user associations (módulos) have features that differ from those of, also self-governed, traditional irrigation organizations. The first objective of this article is to characterize distinctive features. The second objective is to explore, in the módulos, the presence of informal local organizations. The comparative method (between types of organizations and between módulos) as well as the ethnographic method (at the módulos) is used. The results encompass characterization of distinctive features and fact based determination of presence of local organizations in the módulos. The case studies of módulos, in relation to the universe of irrigation districts, are limited. However, the approach is novel, few studies contrast organizational types and there are few ethnographies from the local viewpoint for the módulos. It is shown that there are substantive differences between the organizational types, and that despite the rigidity and centralization of the organizational design of the módulos, there is local organizational effervescence.

Keywords: Mexico; water user associations; irrigation districts; bureaucratic administration; local organizations.
\end{abstract}

Cómo citar: Palerm Viqueira, J. (2020). Caracterización de los módulos de los distritos de riego y presencia de organizaciones locales. región y sociedad, 32, el 335. doi: I0.22198/rys2020/32/1335

* Colegio de Posgraduados, Campus Montecillo. Carretera México-Texcoco km 36.5, C. P. 56230, Montecillo, Texcoco, Estado de México, México. Correo electrónico: jacinta.palerm@gmail.com

Recibido: 26 de abril de 2020

Aceptado: 29 de julio de 2020

Liberado: 25 de agosto de 2020

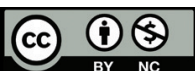

Esta obra está protegida bajo una Licencia Creative Commons Atribución-No Comercial 4.0 Internacional. 


\section{Introducción}

La transferencia de los distritos de riego a los usuarios en la década de 1990 creó nuevos espacios hidráulicos de autogobierno: los módulos de riego, que tienen características distintas a los regadíos vernáculos (regadíos que no son distritos de riego). Después de 30 años ha quedado claro que las diferencias no se relacionan con la novedad de la transferencia y que, en muchos casos, tampoco pueden atribuirse al tamaño de los sistemas de riego. Las diferencias más notables se encuentran en la centralización y en la contratación de personal en cadena de mando, lo cual da a la gestión un carácter burocrático, así como en los criterios de distribución del agua y pago de la cuota de servicio de riego, que implican acceso a volúmenes de agua y pagos diferenciados entre usuarios por la misma superficie de riego.

En la primera parte del texto se presentan algunas características de los módulos de riego en contraste con los regadíos vernáculos. Con ello se pretende señalar las diferencias entre los dos tipos de organizaciones de regadío, a pesar de que ambos tienen autogobierno. Una de las diferencias que en la presente investigación se destacan es la ausencia de organizaciones locales formales en los módulos de riego; en éstos las acciones del regante parecen limitadas a elegir, a través de su representante, a un comité directivo y a realizar los trámites requeridos para tener acceso al agua.

No obstante, hay autores que señalan que aún en la gestión de tipo burocrática las organizaciones, los conocimientos sobre la gestión del agua y las movilizaciones locales desempeñan un papel importante: Freeman Bhandarkar, Shinn, J. Wilkins-Wells y P. Wilkins-Wells (1989) indican las ventajas de las organizaciones locales (a las que llaman organizaciones intermedias) que enlazan al regante con los empleados y las autoridades de la institución a cargo de la gestión del agua (véanse también Freeman y Lowdermilk, 1985; Romero-López y Palerm, 2018). Glick (1972) señala que la burocracia del Estado se apoya en las organizaciones y en los conocimientos locales. Mikhail (2019) aporta evidencia de cómo la población local, obviando las autoridades burocráticas locales, puede movilizar a la burocracia distante. Wittfogel (1966) $)^{1}$ señala que hay límites (rendimientos decrecientes) a la presencia de la burocracia que conducen al desarrollo de espacios de autogestión (comunidades), y acuña el término de democracia de mendigos.

Las preguntas que aquí se plantean son las siguientes: si en los módulos de riego, no obstante su ausencia formal, ¿hay presencia de organizaciones locales encargadas del agua?, ¿la existencia de organizaciones locales coadyuva a una mejor gestión del agua? y ¿cuál es la preferencia social de los regantes minifundistas?

En la segunda parte del texto se proponen algunas estrategias para abordar la etnografía de lo que hace el regante para tener acceso al agua. La intención

1 En contraparte, otros autores han señalado cómo la expansión de la burocracia socava y debilita las organizaciones locales (Oré, 2005a y 2005b; Palerm, 2009a) y consideran importante un marco jurídico de larga duración para dar aliento a las organizaciones autogestionadas (Giménez Casalduero y Palerm, 2007; Ostrom, 2011; Palerm, 2014; Sengupta, 2002). 
es visualizar la importancia de la ausencia o de la presencia de lo local (organización, conocimiento, movilización) en la gestión del agua de riego.

Frente a lo oficial y uniforme, son necesarias estrategias que aborden lo vernáculo, real y plural. Como señala Grossi (1981): "A nivel de la infraestructura de la praxis fue inevitable que comparaciones y aun disensos apareciesen entre la historia oficial y la historia vulgar, entre la nación oficial y la real, entre la unidad hallada en modelos y la pluralidad encontrada en la práctica” (p. 131) (traducción propia). ${ }^{2}$

En la etnografía, desde la perspectiva del regante, se propone abordar cómo accede al agua -incluido cuando lo hace infringiendo la normativa-, cómo se negocia en caso de conflicto y cómo se limita y se sanciona a los que infringen la normativa oficial. También se propone abordar la interacción del regante con el aparato administrativo del módulo.

Algunos resultados preliminares muestran la importancia de las organizaciones locales: a) para mejorar la distribución del agua (Montes de Oca y Palerm, 2013; Montes de Oca, Palerm y Chávez-Mejía, 2012); b) en las resoluciones satisfactorias de conflictos, a escala local en lugar de involucrar y saturar a los niveles jerárquicos superiores; c) para la movilización con miras a negociar la distribución del agua con las autoridades del distrito de riego o con la Comisión Nacional del Agua (CONAGUA); d) en la preferencia social por la continuidad de la organización local para la distribución del agua (Venado Godínez y Palerm, 2013; Venado Godínez, Tavera Fenollosa y Palerm, 2018).

Los módulos en los que se ha realizado trabajo de campo con observación participante, entrevistas abiertas y recorridos, son Cuautla y Yautepec (Distrito de Riego Morelos), Actopan y Tepatepec (Distrito de Riego Tula), Cuxtepeques (Distrito de Riego Cuxtepeques), Tepetitlán (Distrito de Riego Estado de MéxiCo), San Jacinto y Nazas (Distrito de Riego La Laguna), Huapango (Distrito de Riego Arroyo Zarco), módulos del Distrito de Riego Zamora, módulos del Distrito de Riego del río Mayo y Joachín (Distrito de Riego Río Blanco).

\section{Primera parte. Caracterización de los módulos y contrastes con los regadíos vernáculos \\ Módulos de riego: diseño organizativo y base material}

Los módulos de riego son subdivisiones de los distritos de riego y el resultado de la transferencia o entrega - parcial- de la administración a los regantes. El Estado, a través de la CONAGUA, conservó la administración de las presas y otras infraestructuras importantes. Además, en algunos casos, entregó a los regantes infraestructura adicional bajo la figura de sociedad de responsabilidad limitada (S. de R. L.), la S. de R. L. conjunta a los módulos de un distrito. Los módulos, para fines de cantidad disponible de agua, desembalse de las presas y

2 "On the level of the infrastructure of praxis it was inevitable that comparisons perhaps even dissensions should have arisen between official and vulgar history, between the official nation and the real nation, between the unity found in models and the plurality found in practice" (Grossi, 1981, p. 131). 
otros asuntos, cuentan con representación en el comité hidráulico del distrito de riego.

Los módulos y las S. de R. L. pueden considerarse niveles organizativos adicionales en la gestión de los distritos de riego que dan lugar a una cogestión -entre los regantes y el Estado- del espacio hidráulico de dichos distritos.

No obstante hay que tomar en cuenta que la terminología distrito de riego, módulo de riego, entrega de agua en bloque y plan de cultivo esconde a menudo la realidad de cuál es el curso del agua por la infraestructura de presas de almacenamiento, presas derivadoras (azud), canales generales y secundarios o terciarios hasta las parcelas y, por lo tanto, oculta cuáles de los regantes del módulo comparten infraestructura. Aspecto importante, ya que solo entre ellos existe una necesaria solidaridad de base hidrológica.

En efecto, muchos distritos se componen de múltiples sistemas de riego. Incluso hay muchos que ni siquiera comparten una presa común como obra de cabecera. Ello se puede caracterizar como la inexistencia de un traslape entre distrito de riego y sistema de riego, o entre distrito de riego y área de comando de una presa. De tal manera que no hay necesidad de una coordinación técnica a través del espacio del distrito de riego. La centralización en un único distrito de riego de los espacios hidráulicos obedece únicamente a criterios de la administración impuestos por los límites que marca la CONAGUA (Palerm, 2009a). También implica que el tamaño de los sistemas de riego comprendidos en un módulo pueden ser pequeños o medianos, lo que permite una operación en manos de los mismos regantes, sin técnicos (Palerm, 2005 y 2009a).

Por ejemplo, el Distrito de Riego Morelos -que posiblemente corresponde a la situación más frecuente- consiste en pequeños sistemas que toman agua de distintos ríos. Entre los espacios de riego, y por lo tanto entre los regantes del distrito, no hay necesidad de coordinación ni solidaridad de base hidrológica. Los módulos están conformados por los sistemas de riego vertebrados en cada uno de los ríos del distrito. Dichos sistemas comparten, en todo caso, el agua del río -aunque hay también sistemas que dependen de manantiales-. Es decir, únicamente si se requiere coordinación a escala de río, hay requerimiento de cooperación (Gadea-Noguerón, 2019; Palerm, Pimentel-Equihua y Salcedo, 2000).

En otros casos, como en el Distrito de Riego Tula, que tiene una coordinación técnica entre sus distintas fuentes, la subdivisión en módulos se realizó por cercanía geográfica. Así pues, hay módulos que tienen tramos de dos o tres canales generales, con problemáticas distintas y cuya necesidad de coordinación técnica inmediata es con el tramo de canal general aguas arriba, de tal manera que entre los regantes del módulo que dependen de distintos canales generales hay una limitada solidaridad de base hidrológica que se relaciona con las fuentes de agua y con la obra de cabecera del distrito (Venado Godínez y Palerm, 2013).

Lo anterior implica que no existen requerimientos técnicos de coordinación entre todos los espacios hidráulicos del distrito y que no hay solidaridad de base hidrológica entre todos los usuarios. En este sentido, es útil la distinción que hace Vaidyanathan (2009) entre los aspectos funcionales o técnicos y los aspectos políticos de la gestión. El aspecto funcional o técnico se refiere a la coor- 
dinación necesaria; el aspecto político, a la toma de decisiones (pp. 116-117). En principio, las instituciones u organizaciones formales que toman decisiones debieran correlacionarse con los requerimientos técnicos de coordinación.

La transferencia de subdivisiones de los distritos de riego (los módulos) puso de manera formal el gobierno y la administración del espacio hidráulico en manos de los regantes. La ausencia de un traslape entre módulo y sistema de riego implica que la toma de decisiones no siempre responde a las necesidades de coordinación técnica.

El diseño organizativo de los módulos consiste en un comité directivo, elegido en asamblea por los delegados del agua (representantes de los regantes) o a veces en asamblea de todos los regantes. Los delegados del agua son, a su vez, elegidos por los regantes que pertenecen a un mismo ejido o pueblo y que, por lo común, aunque no siempre, comparten la misma infraestructura. Los propietarios privados de mayores dimensiones también eligen a sus propios delegados. El comité directivo contrata personal de oficina y personal para la distribución del agua, y se encarga del mantenimiento, utilizando la maquinaria del módulo o contratando los trabajos necesarios u organizando a los regantes para que, mediante faenas, lo lleven a cabo. Otras tareas consisten en realizar y entregar informes al distrito de riego, participar en el comité hidráulico del distrito y en otras negociaciones hacia el exterior.

El diseño organizativo del módulo es de tipo centralizado, es decir, carece de niveles organizativos formales en su interior, lo cual lo distingue del diseño organizativo que ha sido descrito para sistemas de riego vernáculos y que es, por lo general, descentralizado con múltiples niveles organizativos (o entidades incrustadas o anidadas) (Ostrom, 2011, p. 163; Pimentel-Equihua, 2004; Yoder, 1994a y 1994b). Mientras que un diseño organizativo centralizado favorece una administración burocrática (personal contratado en cadena de mando), la descentralización facilita una operación realizada por los mismos regantes o controlada de cerca por ellos (Palerm, 2006 y 2009b).

Ostrom (2011), en sus principios de diseño para instituciones de recursos de uso común (RUC), que responden a la necesidad o conveniencia de administrar un recurso común a todos los usuarios, señala, como octavo principio, para las organizaciones de mayor tamaño, que "Las actividades de apropiación, provisión, supervisión, aplicación de las normas, resolución de conflictos y gestión se organizan en múltiples niveles de entidades incrustadas" (p. 163). Explica que

El problema que enfrentan los regantes en el nivel de un canal terciario es diferente de los enfrentados por un grupo mayor que comparte un canal secundario. Éstos, a su vez, son distintos de los problemas implicados en la administración de los trabajos fundamentales de desviación que afectan a todo el sistema. (p. 163)

Los niveles organizativos, según los principios de diseño propuestos por Ostrom (2011), implican una fuerte correspondencia entre aspectos funcionales o técnicos de coordinación y aspectos políticos de toma de decisiones. Sin embargo, el diseño organizativo de los módulos, además de carecer de niveles 
organizativos, puede conjuntar espacios hidráulicos entre los cuales no necesariamente se requiere de coordinación funcional o técnica.

Módulos de riego: criterios de distribución de agua

En los regadíos mexicanos (y peruanos) que son distritos de riego es típica la distribución del agua por cultivo, de tal manera que la cantidad de agua que puede recibir el regante varía por hectárea y por cultivo. En la distribución del agua por plan de cultivo y riego, el volumen de agua entregado por hectárea varía para adaptarse al cultivo. El acceso al agua de riego se limita a la tierra de riego del distrito.

En los regadíos de México que no son distritos de riego y en regadíos autogestionados de muchas partes del mundo, lo común es que el agua se distribuya por tanda (o proporcional). Cada regante recibe de forma periódica una cantidad de agua proporcional a su tierra de riego. A su vez, cada regante contribuye al mantenimiento del sistema de riego con faenas y cuotas proporcionales a su tierra de riego. Esta distinción es una apreciación atribuible a Trawick (2001, 2002 y 2003).

En los casos de sistemas con distribución por tanda (o proporcional), todos reciben el mismo volumen de agua por hectárea y los regantes mismos deciden en qué cultivos hacer uso de ella. "El manejo de la distribución y programación del riego está basado en la tanda o turno sin considerar el cálculo de la demanda hídrica del cultivo" (Pérez Magaña, Macías López y Gutiérrez Villalpando, 2019 , p. 10). En caso de escasez, hay una distribución proporcional del agua existente (Rodríguez Meza, 2000, pp. 374-375; Villarreal y Manzanal, 2011, p. 40). Incluso para lograr la equidad en la distribución, hay estrategias para rotar entre años los campos en los cuales da inicio el riego, o los campos dedicados a cultivos con mayor demanda de agua (Palerm, 1999 y 2001; Palerm, Pimentel-Equihua y Salcedo, 2000, pp. 81-82).

La distribución por tanda tiene flexibilidad mediante mecanismos de otorgar agua en caso de riesgo del cultivo o de realizar intercambios y venta de tandas de agua $^{3}$ (Palerm, 1999, 2001 y 2003; Pérez Magaña, Macías López y Gutiérrez Villalpando, 2019). Otra estrategia de flexibilidad consiste en almacenar de manera temporal la tanda de agua en jagüeyes o pequeños almacenamientos a nivel de parcela y poder regar a conveniencia (Palerm, 2003; Vaidyanathan, 2009, pp. 116-117).

3 La legislación del siglo XX en México no necesariamente ha permitido los intercambios o venta de la tanda de agua de riego, mucho menos regar fuera de la superficie de las tierras de riego. En varios reglamentos de sistemas se señala que "cuando alguna toma no esté en aptitud de recibir las aguas que le correspondan, o que los usuarios de ella las rehúsen en todo su volumen o en parte de él, se considerará, para los efectos de la distribución, que la toma de que se trate ha recibido el total de las aguas que le correspondía, quedando por lo tanto, el volumen no recibido, disponible para ser distribuido entre las demás tomas" (Gutiérrez Rivas, Romero-López y Palerm, 2017, pp. 218, 289 y 411). En el caso de las aguas de pozo o de galería de libre alumbramiento y, por lo tanto, no sujetas a concesión (aunque en la actualidad, según la legislación vigente, están sujetas a concesión), podían aplicarse o venderse a criterio del propietario o socio del pozo o galería; tampoco estaban limitadas a regar superficies predeterminadas. 
En el caso de regadíos con pozos o de regadíos con galerías filtrantes, autogestionados, a cada socio del pozo o de la galería le corresponde una cantidad (volumétrica) del agua de la que puede hacer uso o la puede vender. El acceso se realiza por tanda y la venta puede ser de todo el ciclo o de sólo una tanda (Campos, Henao, Enge y Whiteford, 2000; Mestries y Bonilla, 2010).

La distribución proporcional del agua por cantidad de tierra en tandeos parejos permite una mayor transparencia en la distribución y en las obligaciones de mantenimiento, ya que éstas son también proporcionales a la cantidad de tierra de riego (Trawick, 2001, p. 367). La distribución proporcional está aunada con frecuencia al control y a la supervisión locales (Boelens y Vos, 2012, p. 23). El regante tiene incentivos para mejorar la eficiencia del riego, ya que el uso más o menos eficiente de su volumen de agua redunda en sus cultivos (Roa García, 2014, p. 308; Trawick, 2003, p. 989).

En comparación con la distribución del agua por plan de cultivo y riego, la distribución proporcional es más transparente, más equitativa y tiene incentivos para mejorar la eficiencia de riego. En el caso de Perú las organizaciones que utilizan el sistema de distribución por plan de riego y cultivo gastan más en personal capacitado para realizar los cálculos que las organizaciones que distribuyen de forma proporcional (Lajaunie, Tillier, Serrano Navarro, Lombana Córdoba, Scheierling y Chinarro, 2013, p. 86; Roa García, 2014, pp. 308-309; véase también Boelens y Vos, 2012, p. 22).

En los regadíos mexicanos que son distritos de riego es más común la distribución por cultivo, de tal manera que la cantidad de agua que puede recibir el regante varía por hectárea y por cultivo. Es decir, el volumen de agua entregado por hectárea varía para adaptarse al cultivo; sin embargo, está limitado a la tierra considerada de riego. La decisión sobre cultivos la precede la determinación de cantidad de agua disponible en un año dado, lo cual es particularmente pertinente en el caso de las presas de almacenamiento. La determinación la realiza la CONAGUA, y el comité hidráulico (conformado por el personal técnico de ésta y los representantes de los módulos y de la S. de R. L., si existe) propone la superficie destinada a determinados cultivos y la cantidad de agua que se utilizará por mes (Pedroza González e Hinojosa Cuéllar, 2014, pp. 11, 19-20).

La implementación de la distribución del agua por los planes de riego y cultivo, en México, según Romero Blake (2008) inicia con una reglamentación de 1953 sobre la conformación de comités directivos en los distritos de riego. "Los comités directivos [de los distritos de riego] presentaban varios programas de cultivo a escoger para cada temporada agrícola, y una vez elegido uno, cada agricultor recibe sólo el agua necesaria para cumplir su parte de ese programa en el momento preciso en que los cultivos escogidos necesitan riego" (p. 50). Es posible que el agua de riego se distribuya de manera desigual en los distritos de riego desde cuando menos 1946 con la Ley de Riegos, en cuyo artículo 31 se mencionan los "calendarios de cultivo y de riego" y que fue publicada en el Diario Oficial de la Federación (DOF) el 31 de diciembre de ese año (Lanz Cárdenas, 1982) y sigue vigente en la Ley de Aguas Nacionales, como señala el artículo 69bis: 
Los usuarios de los distritos de riego deberán respetar los programas de riego determinados conforme a la disponibilidad del agua para cada ciclo agrícola. La realización de siembras no comprendidas en los programas de riego y de siembra que para tal fin hubieren aprobado las autoridades competentes para ese ciclo agrícola, originará la suspensión del derecho a contar con el servicio de riego, aun cuando existan cultivos en pie. (CONAGUA, 2012, p. 86)

En las décadas de 1940 y 1950 la Secretaría de Recursos Hidráulicos debía coordinarse con la Secretaría de Agricultura y Ganadería sobre los planes de cultivo en los distritos de riego (por ejemplo, "Acuerdo que crea dentro de la Secretaría de Agricultura y Ganadería, la Dirección General de Planeación Agrícola en los Distritos Nacionales de Riego" (DOF, 25 de abril de 1951; Lanz Cárdenas, 1982). En la actualidad, el plan de riego de los módulos debe ser aprobado por la CONAGUA (Pedroza González y Hinojosa Cuéllar, 2014, pp. 10 y 19-20).

Como ya se ha señalado, el agua de riego se distribuye de manera desigual en los distritos de riego. Esto es, los derechos de riego no implican que aquellos dados de alta en el padrón de usuarios tengan acceso a una cantidad proporcional de agua conforme a su superficie de riego. El agua de riego no se distribuye de manera proporcional entre la tierra de riego, sino que el agua se distribuye conforme al cultivo. Ello es un reto para el cálculo de cuotas y contribuciones para la operación y el mantenimiento de la infraestructura hidráulica. En el caso de la distribución proporcional de agua de riego por hectárea, típica de los regadíos vernáculos, las cuotas y las contribuciones se calculan también de manera proporcional.

Sistemas de cálculo para la cuota de servicio de riego (o formas de cobro)

Las soluciones, de acuerdo con Espinosa (1962, pp. 516-519), que se dieron en los distritos se presentan a continuación, cabe considerar que el agua de riego siempre se entrega conforme al cultivo (excepto quizá en el último caso), lo que varía es el cálculo de la cuota.

Tipo 1. Por hectárea y por riego. Se paga por riego sin importar el cultivo y la cantidad de agua que se use en cada riego. Según Pimentel Equihua (2020), en algunos módulos se paga por riego y lo que está establecido es el número de éstos por cultivo. Por ejemplo, en los módulos del Distrito de Riego Zamora, para la fresa son 40 riegos; para el trigo, 4 riegos, aunque el volumen de agua para el riego de fresa y el de trigo es distinto, así como la cantidad de agua para el primer riego del trigo y los subsiguientes. Según Espinosa, este cálculo de la cuota era muy usado y exige mucha labor de contabilidad.

Tipo 2. Por hectárea regada al año. Se paga sin importar el cultivo ni la cantidad de agua. Espinosa reporta que se usaba en pocos distritos.

Tipo 3. Por hectárea regable al año. El pago no depende del cultivo ni de la cantidad de agua; también están obligados aquéllos que no riegan. Espinosa reporta que se usaba en muchos distritos y que es fácil de manejar, aunque propicia el desperdicio de agua. 
Tipo 4. Por hectárea y por cultivo. Se paga por hectárea regada, pero la cuota es diferente para cada cultivo; parece que se considera el distinto volumen por riego para distintos cultivos. Espinosa reporta que se utiliza en muy pocos distritos, pero que es más equitativo (suponiendo la equidad como el mayor acercamiento al pago igual por volumen de agua).

Tipo 5. Volumétrico. Se paga por millar de metros cúbicos servidos, sin tener en cuenta la superficie ni el cultivo. Espinosa señala que se utilizaba en los distritos de San Juan del Río, Arroyo Zarco, Río Altar, Río Yaqui, y que, en estos casos, el agricultor debe solicitar y pagar el volumen que crea vaya a utilizar. Es una forma de cuota que requiere aforadores que midan el gasto de entrada a la regadera de cada agricultor. Pero, según señala Espinosa, es la forma de cuota más equitativa (suponiendo la equidad como el pago igual por volumen de agua) y es la única que obliga al agricultor a usar las menores cantidades de agua (para pagar menos).

Todas estas soluciones o variantes siguen vigentes en los módulos. En un texto más reciente, se reportan los siguientes sistemas de cálculo. Tipo 1: por hectárea riego. Tipo 2: por hectárea regada al año. Tipo 4: por hectárea y tipo de cultivo y, lo que parece ser una variante (tipo 4-b), por hectárea y tipo de demanda de agua del cultivo; es decir, de alta demanda, de demanda media y de baja demanda. Tipo 5: volumétrico con distintas formas de medición (tipo 5a: por millar de metros cúbicos; tipo 5b: volumétrico por litro por segundo por hectárea en 24 horas; y tipo 5c: por hora con cierto gasto en el caso de bombeos (Palacios Vélez, Exebio García, Mejía Sáenz, Santos Hernández y Delgadillo Piñón, 2002, p. 511). Según el mismo texto, en los distritos transferidos se presenta el tipo 1 (hectárea riego) en $8.7 \%$ de los casos; los tipos 2 y 4 (ha/ anual y ha/cultivo) en 48.3\%; y el tipo 5, en 43.0\% (Palacios Vélez, Exebio García, Mejía Sáenz, Santos Hernández y Delgadillo Piñón, 2002, p. 508, figura 2).

Las soluciones anteriores suponen la autorización de cultivo/cantidad de agua y, por lo tanto, un procedimiento para dar dicha autorización, a no ser que cualquier cultivo/cantidad de agua se pueda autorizar.

De igual forma, todas las soluciones requieren un pago monetario de parte de los regantes. Cabe señalar que en regadíos de reparto proporcional es frecuente que el mantenimiento se realice con faenas (o fatigas) de los mismos regantes de manera proporcional en la infraestructura que los beneficia. Los mismos regantes realizan la operación bajo cargos honoríficos, de tal manera que no hay pago de cuota de riego o es muy pequeño. Si se comparan los regadíos vernáculos con los distritos de riego, resulta que en los distritos los agricultores con mayor capacidad económica son los que pueden pagar con mayor facilidad los riegos para los cultivos cuyo requerimiento de agua es mayor; es el caso de los tipos de cuota 1,4 y 5 .

El cálculo de cuotas en los módulos debe considerarse bajo la luz de la propuesta de los principios de diseño de instituciones de larga duración que propone Ostrom (2011), en específico el segundo punto que señala:

Coherencia entre las reglas de apropiación y provisión con las condiciones locales. Las reglas de apropiación que restringen el tiempo, el lugar, 
la tecnología y la cantidad de unidades de recurso se relacionan con las condiciones locales y con las reglas de provisión que exigen trabajo, material y dinero o ambos. (p. 148)

Sin embargo, en el caso de los módulos, la coherencia entre las reglas de apropiación (el agua de riego) y la provisión (la cuota por servicio de riego) parece poco transparente. La coherencia se vuelve aún más dudosa cuando el espacio hidráulico que gobierna un módulo corresponde a los espacios entre los cuales no hay solidaridad de base hidrológica.

La relación entre las reglas de apropiación y la provisión en el proceso de transferencia no parece haber sido motivo de reflexión más allá de evitar el subsidio a los distritos de riego y asegurar la conservación y el mantenimiento. De ahí la insistencia, incluso en la legislación, en la cuota de autosuficiencia (CONAGUA, 2012; Palacios Vélez et al., 2002; Trava Manzanilla, 1995).

Mercado de agua y mercado de derechos de agua

El mercado del agua tiene también características distintas en los distritos y los regadíos vernáculos. En el caso de los regadíos vernáculos, es posible vender las tandas de agua o rentar el acceso al agua de una cierta tierra de riego, mientras que en los distritos de riego de México (y de Perú), aunque hay un supuesto mercado del agua, éste, en realidad, consiste en la renta o la venta de los derechos de agua (Fortis-Hernández y Alhers, 1999; Romero Blake, 2008, pp. 32 y 112). El regante puede rentar su derecho de agua, pero la cantidad de agua que reciba el arrendatario depende del cultivo.

No obstante, Zegarra (2004) señala, que un mercado de agua, donde se pueden realizar reasignaciones por la vía de dicho mercado, implica "eliminar la noción de que el acceso al agua depende de un plan de cultivo y riego o de lo que el agricultor desea sembrar. El paso de este concepto al de que cada usuario tiene una dotación fija por hectárea por año (por ejemplo, proporcional a la oferta disponible ese año)..." (p. 60). La conceptualización de mercado de agua de la que habla Zegarra corresponde al caso de los regadíos mexicanos que no son distritos de riego, donde se pueden vender o intercambiar tandas de agua.

En México, en particular en los distritos de riego del norte, la posibilidad de renta y de la compraventa de derechos de agua ha llevado a un desplazamiento muy importante de los pequeños agricultores, que han sido reemplazados por los arrendatarios (llamados rentistas) que encabezan grandes explotaciones agrícolas (Fortis-Hernández y Alhers, 1999; Torregrosa y Salgado, 2018).

El fenómeno del rentismo, como se denomina en México, es decir la práctica extendida de rentar los derechos de agua, ha desplazado a los derechohabientes originales, que han abandonado la producción agrícola, de tal manera que el tipo de productores ha cambiado. Esa práctica impide evaluar si la introducción del autogobierno a los distritos de riego ha permitido mejorar la gestión del agua y por lo tanto mejorar la producción agrícola, porque derechohabientes y productores ya no coinciden. 


\section{Segunda parte. Estrategias y ejemplificación: etnografía desde la perspectiva del regante}

En este apartado se aborda la etnografía de la resolución de las tareas esenciales del sistema de riego, no desde la perspectiva de la organización módulo, sino desde la perspectiva del regante (derechohabiente o arrendatario). Esto es, dar seguimiento a las acciones del regante para tener acceso al agua de riego. ${ }^{4}$

En la gestión burocrática de espacios hidráulicos, el regante más bien realiza trámites para tener acceso al agua y el aparato administrativo y de gobierno, además de operar canales y supervisar el mantenimiento, registrar información. En la estructura formal del módulo de riego no existen organizaciones intermedias. Se proponen aquí varios hilos a los que se puede dar seguimiento.

\section{La distribución del agua}

En primer lugar, la distribución del agua: la etnografía de cómo el regante logra que el agua llegue a su parcela en cantidad y oportunidad. La interacción entre el regante y el módulo para que aquél pueda tener acceso al agua en una temporada dada, inicia con el papeleo que debe realizar; por ejemplo: solicitar la autorización del cultivo; su otorgamiento; hacer el pago de riegos (una forma frecuente de pago de la cuota de servicio de riego) en la oficina recaudadora; esperar la entrega de un vale de riego (vale que debe ir, por lo regular, firmado por el delegado del agua y que avala que los canales de acceso están limpios); solicitar el riego al canalero, quien -con los vales- debe establecer el orden de riego (para el regante, el orden de riego puede ser muy importante dada la oportunidad del riego o por estrés hídrico del cultivo); y, por fin, se lleva a cabo la entrega de agua.

La interacción entre el regante y el módulo para concretar el acceso al riego debe finalizar con la entrega de agua que, en principio, realizan los canaleros que contrata el comité directivo del módulo y que deben seguir las instrucciones de su cadena de mando.

Es conveniente observar la entrega de agua desde dos ángulos: por una parte, cómo se da de hecho esa entrega; es decir, cuál es la etnografía de cómo toma el agua el regante. Por otra, qué es lo que hace el canalero, por ejemplo, las tareas de operación que realiza y cuál es, si es el caso, la interacción entre éste y el regante. Cabe señalar que en el acceso al riego hay que considerar también la toma de agua por el regante y la entrega de agua por el canalero fuera de la normativa.

4 Por supuesto, es pertinente considerar el contexto del sistema de riego como espacio hidráulico y del módulo como espacio administrado, espacios que tal vez no se traslapan. Así como el tamaño del sistema o de los sistemas de riego, por la posible complejidad (Hunt, 1997; Palerm, 2001). Es también pertinente la descripción de las fronteras en el espacio hidráulico entre módulo y organización local, es decir dónde se realizan las acciones del módulo y las de la organización local. Las fronteras pueden variar si se visualiza el mantenimiento o la distribución del agua, o por las épocas del año, como el estiaje (Hunt, 1997; Palerm, 2001; Palerm, Martínez-Saldaña y Escobedo, 2000). En las descripciones que siguen se prescinde de este contexto que no se debe omitir en el trabajo de campo. 
Se puede ejemplificar con algunos casos que no corresponden a lo que podríamos suponer es la norma: el regante le entrega al canalero su vale y con ello solicita el riego, el canalero a su vez organiza la entrega de agua y se la provee de manera directa o le señala cuándo puede tomarla. Los casos que presentamos, posiblemente anómalos, en su mayor parte se refieren a la presencia de organizaciones intermedias entre el canalero y el regante, es decir, el canalero no entrega el agua al regante, sino que entrega a una organización local que a su vez hace entrega al regante.

1) En el caso de los módulos del Distrito de Riego Zamora, cada regante toma el agua cuando lo requiere su cultivo. El canalero tiene algunas actividades de operación (como mantener el tirante del canal que tiene tomas directas del río) y realiza recorridos de vista con el fin de hacer un reporte de los cultivos en pie. Dicho reporte es la base para el cobro de la cuota de servicio de riego (Pimentel Equihua, 2020).

2) En el caso de los módulos Nazas y San Jacinto en el alto Nazas del Distrito de Riego de La Laguna, hay continuidad de los distribuidores del agua que existían antes de la transferencia. A éstos se les llama regidores y han sido nombrados de entre los regantes y por ellos mismos. Los regidores han sido, en algunos casos, reconocidos por el módulo correspondiente como personal de operación y reciben un salario por parte de éste. Únicamente en el periodo en que las aguas están controladas por el distrito, la entrega de agua en bloque la realiza personal de la CONAGUA en la bocatoma de los pequeños sistemas de riego ubicados en el espacio administrado por el módulo (Cháirez y Palerm, 2013a y 2013b).

3) En el caso del módulo Huapango del Distrito de Riego de Arroyo Zarco, a los delegados de agua se les conoce como representantes y dan continuidad a los distribuidores del agua preexistentes a la transferencia. Se encargan de recibir el agua entregada por los canaleros y de distribuirla a los regantes. Los representantes son nombrados por los mismos regantes y, con frecuencia, de entre ellos mismos. De esta manera los delegados del agua, de hecho, son personal de operación. Los regantes pagan de forma directa la retribución que reciben los representantes. Éstos tienen a su cargo el cobro de riego que hacen llegar al comité directivo (Montes de Oca, 2020).

4 y 5) En los módulos Tepatepec y Actopan del Distrito de Riego Tula hay distribuidores del agua llamados zanjeros, que existen desde antes de la transferencia y que son nombrados de entre los regantes y por ellos mismos. Los zanjeros, junto con los regantes con vales, deciden el orden de riego. Los zanjeros y los regantes vigilan que se respete el turno establecido. Los canaleros no pueden entregar agua adicional o en turnos especiales cuando el turno lo establecen los mismos regantes, es de conocimiento público y está bajo la vigilancia de los zanjeros y de los regantes (Galindo-Escamilla, Bárcenas y Palerm, 2018, 2019; Venado Godínez y Palerm, 2013; Venado Godínez et al., 2018).

4) En el caso del módulo Tepatepec del Distrito de Riego Tula -que en la actualidad está en situación de no entregado y cuya operación la realiza un equipo contratado por la CONAGUA-, la política del módulo de suprimir a los zanjeros ha provocado una desobediencia generalizada de respetar el orden de 
riego de los canaleros. El canalero no puede hacer cumplir el orden de riego. Los zanjeros, junto con los regantes, establecían el orden de riego entre los que habían presentado su vale. Los regantes han presionado para conservar a los zanjeros, así como a los canaleros que ellos mismos habían seleccionado (Venado Godínez, Tavera Fenollosa y Palerm, 2018).

Las quejas de los regantes sobre el equipo de trabajo contratado por la CONAGUA ha sido la inflexibilidad en el orden de riego, así como la falta de capacidad de supervisión de los regantes de las entregas de agua realizadas por el canalero en la medida que la ausencia de supervisión permite que el canalero asigne turnos preferenciales o entregas adicionales de agua.

5) En el caso del módulo Actopan del Distrito de Riego Tula, los delegados de agua son conocidos como representantes, y tal cargo lo desempeñan los presidentes de los comisariados ejidales o los representantes de los propietarios privados minifundistas de las comunidades. Los representantes están integrados a la mesa directiva del módulo, la cual se reúne de manera semanal; además, supervisan, junto con los zanjeros, la entrega de agua en las compuertas de los canales generales. Los canaleros son contratados o despedidos a sugerencia de los regantes, quienes, por lo demás, están sujetos a justificar su trabajo en las reuniones semanales de los representantes de los ejidos y de los propietarios privados minifundistas. También están sujetos a la supervisión directa de los representantes de cada compuerta de los canales generales (Galindo-Escamilla, Bárcenas y Palerm, 2018 y 2019).

6) En el caso del módulo Cuautla, así como en lo que iba a ser el módulo Yautepec (en la actualidad desincorporado del distrito debido a que rechazó la transferencia) del Distrito de Riego Morelos, hay continuidad, de facto aunque no de jure, de las autoridades y de los operadores locales: aguadores, jueces de agua, guardatierras, fontaneros; y únicamente en los sistemas más grandes del módulo Cuautla hay preseros y canaleros contratados por dicho módulo. En ambos casos ha habido conatos de desplazar a las autoridades y a los operadores locales y de centralizar la gestión (Ávalos, Aguilar Sánchez y Palerm, 2010; Gadea-Noguerón, 2019; Palerm, Rodríguez-Haro, López Pacheco, Cháirez, Morán y Osorio, 2001).

7) En el caso del módulo y Distrito de Riego Cuxtepeques, en algunos espacios los regantes prefieren hacerse cargo de la distribución en época de estiaje (Zenteno, 2020).

El papel de los delegados del agua para coadyuvar a la distribución local

En ausencia de organizaciones intermedias, los requerimientos de coordinación técnica o funcional, así como de toma de decisiones que corresponden a espacios hidráulicos particulares al interior del módulo (que comparten infraestructura y entre los cuales hay una necesaria solidaridad de base hidrológica), posiblemente puedan expresarse a la mesa directiva o al personal de operación del módulo, a través de los delegados del agua.

Ello es posible cuando los regantes del mismo pueblo o ejido reciben el agua para sus parcelas mediante la misma infraestructura, dado que los delegados 
del agua son electos por los regantes ejidales o por regantes de propiedad privada minifundista de un ejido o pueblo.

Los delegados eligen en asamblea a la mesa directiva y deciden los montos de las cuotas de riego. Sobre lo que se tienen menos noticias es si son un canal de trasmisión de información, quejas o conflictos.

En los módulos, en algunos casos, se ha reforzado o se ha suplantado (ambas palabras cargadas de significado) al delegado de agua al otorgar el cargo al comisariado ejidal, a las autoridades o a los encargados de distribuir el agua preexistentes a la transferencia. Éste es el caso de los representantes en el módulo Huapango del Distrito de Riego Arroyo Zarco. Ahí los encargados de distribuir el agua han ocupado la posición de delegados; en otros módulos y distritos, los regidores, encargados de distribuir el agua, se han integrado como personal de operación. Tales son los casos de los módulos de la parte alta del Nazas del Distrito de Riego de La Laguna. Hay otros casos, como el de los módulos del Distrito de Riego Tula, en los cuales los zanjeros, encargados de distribuir el agua, siguen siendo informales. En todos estos casos, autoridad y encargado de distribuir el agua son sinónimos, tal y como es típico en regadíos vernáculos (Palerm, 2006).

Otro recurso que vale la pena destacar es el del módulo Actopan del Distrito de Riego Tula. Mediante un rediseño organizativo ha solucionado la centralización, pues ha incluido en la mesa directiva a todos los representantes (o delegados del agua), con lo cual ha puesto la representación local en el centro del gobierno del módulo.

El cargo de delegado de agua, conocido como representante, lo desempeñan los presidentes de comisariados ejidales o representantes de propietarios privados minifundistas de comunidades. Dichos representantes están integrados a la mesa directiva del módulo. Ésta se reúne una vez a la semana. Además, supervisan, junto con los zanjeros, la entrega de agua en las compuertas de los canales generales. Hay asuntos que se consideran locales y que deben resolver el representante, el zanjero y los regantes locales. Hay otros asuntos de competencia más amplia que se tratan en el comité directivo. En todos los casos el o los canaleros deben responder a las inquietudes de los regantes (Galindo-Escamilla, Bárcenas y Palerm, 2018 y 2019).

El mantenimiento: el ingreso de cuotas por servicio de riego

El ingreso de cuotas por el servicio de riego es el que comúnmente utilizan los módulos para sufragar el mantenimiento de la infraestructura. Ello trae aparejados dos problemas: el primero, la forma en que se calcula la cuota de riego con su consecuente - poca- transparencia, así como la -dudosa-congruencia entre apropiación y provisión; el segundo, la posible falta de traslape entre el espacio del módulo y el espacio de la infraestructura en común (un sistema de riego).

El sistema de cálculo de la cuota de servicio de riego varía entre los distritos, y la mayoría de los tipos de cálculo no son proporcionales a la superficie que se riega. La poca transparencia y la falta de congruencia pueden tener efectos 
en la disposición del pago, aunque cabe mencionar que en el módulo Joachín del Distrito de Riego Río Blanco parece ser más transparente, pues se paga una cuota por hectárea regable (tipo 3), que se subdivide en cuota de riego y en cuota para mantenimiento; además, se paga una cuota por cultivo (tipo 4) (Salomón, 2020).

Cuando el módulo realiza el mantenimiento y hay varios sistemas de riego, los regantes pueden quejarse si no tienen la seguridad de que el mantenimiento ha sido realizado en la infraestructura que los afecta. Por ejemplo, es motivo de inquietud en algunos módulos del Distrito de Riego Zamora cuando no tienen la certeza de que sus pagos de cuota por servicio de riego hayan sido aplicados en el mantenimiento de sus canales (Pimentel Equihua, 2020).

Por otra parte, en los módulos no es raro que parte del mantenimiento lo realicen los mismos regantes mediante faenas (o fatigas). Al parecer, en estos casos, la inversión de trabajo se efectúa sobre la obra hidráulica que les afecta de forma directa y el aporte de cada regante es proporcional a su tierra de riego. En este aspecto, se siguen los mismos criterios que en los regadíos vernáculos, donde todos los regantes participan de manera proporcional a su tierra de riego en el mantenimiento de la infraestructura que los afecta. Lo que es curioso es que si el mantenimiento se paga con cuotas, puede haber una participación (monetaria) diferencial por hectárea entre los regantes, ya que dicha participación depende de cómo se calcule la cuota por servicio de riego.

Los espacios de mantenimiento a cargo de los regantes pueden ser de los canales menores o pueden abarcar todo el sistema de riego. La organización de las faenas y el seguimiento del cumplimiento se realizan desde la base. Sin embargo, en algunos casos se reporta el cumplimiento al módulo, por ejemplo, en los módulos Actopan y Tepatepec del Distrito de Riego Tula, el delegado firma el vale de riego que acredita el cumplimiento del mantenimiento realizado a los canales secundarios o terciarios (Galindo-Escamilla, Bárcenas y Palerm, 2018 y 2019). Ello confiere al delegado funciones administrativas, que son distintas y adicionales a las de representación.

Se ha reportado la participación directa de los regantes con faenas, además de en los módulos del Distrito de Riego Tula, en el módulo Cuautla del Distrito de Riego Morelos (Ávalos Aguilar y Palerm, 2010; Palerm et al., 2001), en el del Distrito de Riego Cuxtepeques (Zenteno, 2020), en el de Joachím del Distrito de Riego Río Blanco (Salomón, 2020), en los de Nazas y San Jacinto del Distrito de Riego La Laguna (Cháirez y Palerm, 2013a y 2013b), en el de Huapango del Distrito de Riego Arroyo Zarco (Montes de Oca, 2020) y en aquellos módulos que no aceptaron la transferencia y fueron desincorporados de su respectivo distrito: Yautepec del Distrito de Riego Morelos y Tepetitlán del Distrito de Riego Estado de México (Gadea-Noguerón, 2019; Montes de Oca, Palerm y Chávez-Mejía, 2010; Montes de Oca, Palerm y Chávez-Mejía, 2012). Los espacios de participación directa de los regantes para mantenimiento no necesariamente coinciden con los de distribución del agua.

Además de la cuota por servicio de riego y de la inversión directa de trabajo, en algunos casos otra contribución al mantenimiento puede provenir de los municipios. Los módulos también tienen otras fuentes de ingreso que pueden 
destinarse al mantenimiento, como son la renta de maquinaria y los subsidios federales por vía de la CONAGUA (Pimentel Equihua, 2020; Zenteno, 2020).

Las movilizaciones

Las movilizaciones de los regantes para presentar y negociar con los niveles jerárquicos superiores la solución a problemas que no ha podido negociarse en el nivel local, prescinden en general de las estructuras formales. Ello es típico de los módulos de la parte baja del Distrito de Riego Tula (Venado Godínez y Palerm, 2013).

\section{Conclusiones}

En la primera parte se presentaron las diferencias entre los módulos y los regadíos vernáculos. Una de esas diferencias consiste en que aquéllos tienen un diseño organizativo centralizado y la contratación del personal en cadena de mando. En principio, este diseño, a diferencia del diseño organizativo en los regadíos vernáculos, no favorece la existencia de organizaciones locales. No obstante, como se expuso en la segunda parte de este texto, hay, en los módulos de riego estudiados, una importante presencia de organizaciones locales que atienden la distribución del agua y el mantenimiento. El mantenimiento, basado en la participación directa de los regantes con faenas y organizado y supervisado localmente, pasa inadvertido a pesar de ser una expresión de organización local, probablemente debido a que es observable solo durante los cortos periodos en que se realizan las faenas.

Las organizaciones locales, al menos aquellas que en la actualidad existen en los módulos, se fundaron en muchos casos desde antes de la transferencia -al parecer, también con el carácter de informales-. Sin embargo el diseño organizativo de los módulos no consideró integrar las organizaciones locales ya existentes. Incluso en algunos casos el módulo parece haberse esforzado en eliminar a las organizaciones locales. No obstante, en otros casos, los cargos de las organizaciones locales preexistentes a la transferencia han sido integrados o asimilados a la nueva estructura formal del módulo como personal de operación o de gobierno, con lo cual se gana formalidad.

En el balance de si ha aumentado o disminuido la presencia de las organizaciones locales, es posible señalar la resistencia de éstas a desaparecer (como en el caso de los zanjeros del módulo Tepatepec del Distrito de Riego Tula). Además, se observan la integración o la asimilación en la nueva estructura (como en el caso de los representantes del módulo Huapango del Distrito de Riego Arroyo Zarco) y la suma de nuevas acciones (como en el caso del módulo Actopan del Distrito de Riego Tula), ${ }^{5}$ a pesar de que las organizaciones locales no son reconocidas en el diseño organizativo de los módulos.

5 También de interés es el caso de Tepetitlán, en el que las comunidades, debido al retiro de la gestión por el distrito de riego, toman acciones organizativas hacia adentro y hacia afuera de la comunidad que mejoran la distribución del agua (Montes de Oca y Palerm, 2013; Montes de Oca, Palerm y Chávez-Mejía, 2010 y 2012). 
La estrategia metodológica de la mirada etnográfica desde el regante, desde lo local $-\mathrm{y}$ no desde las oficinas centrales del módulo, es decir, desde lo formal- ha permitido visualizar las capacidades organizativas de los regantes. Pero falta aún la etnografía de los tratos del regante con el aparato administrativo del módulo (el papeleo).

Los casos seleccionados corresponden a regadíos ubicados en Mesoamérica con alta presencia de campesinos y de comunidades organizadas. Los distritos de riego del norte siguen una dinámica muy pronunciada de concentración de la producción y de desplazamiento de pequeños agricultores.

La ampliación y la profundización en los estudios de caso permitirán hacer un balance de la importancia de las organizaciones locales en la gestión del agua de riego, en particular, ahí donde la pequeña agricultura no ha sido desplazada. Incluso se puede aventurar que las organizaciones locales contribuyen a que la pequeña agricultura no sea desplazada por arrendatarios que concentran grandes superficies.

\section{Agradecimientos}

Por su lectura y comentarios, a Guillermo Zenteno, Nohora Guzmán, Acela Montes de Oca, María Luisa Torregrosa y, muy en especial, a Ligia Tavera. Igual agradecimiento a los árbitros anónimos.

Con el apoyo financiero del CONACYT, proyecto "Distritos de riego: impacto de los cambios en la legislación sobre las capacidades autogestivas de los regantes", con número de referencia 236867.

\section{Referencias}

Ávalos, C., Aguilar Sánchez, G., y Palerm, J. (2010). Gestión técnica y social del uso del agua en Morelos: caso del río Cuautla. México: Universidad Autónoma Chapingo.

Boelens, R., y Vos, J. (2012). The danger of naturalizing water policy concepts: water productivity and efficiency discourses from field irrigation to virtual water trade. Agricultural Water Management, 108, 16-26.

Campos, F., Henao, L. E., Enge, K., y Whiteford, S. (2000). El valle de Tehuacán. En J. Palerm y T. Martínez Saldaña (eds.), Antología sobre pequeño riego, Vol. Il. Organizaciones autogestivas (pp. 253-344). México: Colegio de Postgraduados y Plaza y Valdés.

Cháirez, C., y Palerm, J. (2013a). Organizaciones autogestivas en los distritos de riego: el caso del módulo II (Nazas) del Distrito de Riego 017. En J. Palerm y T. Martínez-Saldaña (eds.), Antología sobre riego: instituciones para la gestión del agua: vernáculas, alegales e informales (pp. 77-107). México: Biblioteca Básica de Agricultura, Colegio de Postgraduados y Grupo Mundi-Prensa. 
Cháirez, C., y Palerm, J. (2013b). Organizaciones autogestivas en los distritos de riego: el caso del módulo III (San Jacinto) del Distrito de Riego 017. En J. Palerm y T. Martínez-Saldaña (eds.), Antología sobre riego: instituciones para la gestión del agua: vernáculas, alegales e informales (pp. 109-152). México: Biblioteca Básica de Agricultura, Colegio de Postgraduados y Grupo Mundi-Prensa.

Comisión Nacional del Agua (CONAGUA). (2012). Ley de Aguas Nacionales y su Reglamento. Recuperada de http://www.conagua.gob.mx/CONAGUA07/Publicaciones/Publicaciones/SGAA-37-12.pdf

Diario Oficial de la Federación (DOF). (25 de abril de 1951). Acuerdo que crea dentro de la Secretaría de Agricultura y Ganadería, la Dirección General de Planeación Agrícola en los Distritos Nacionales de Riego. Recuperado de http://www.dof.gob.mx/nota_to_imagen_fs.php?codnota=4468999\&fecha $=25 / 04 / 1951$ \&cod_diario $=189284$

Diario Oficial de la Federación (DOF). (31 de diciembre de 1946). Ley de Riegos. Recuperado de http://www.dof.gob.mx/nota_to_imagen_fs.php?cod_diario $=198619$ \&pagina $=13$ \&seccion $=5$

Espinosa Vicente, E. (1962). Los distritos de riego. Su administración, operación y conservación. México: Compañía Editorial Continental.

Fortis-Hernández, M., y Alhers, R. (1999). Naturaleza y extensión del mercado del agua en el D. R. 017 de la Comarca Lagunera, México. México: Instituto Internacional del Manejo del Agua.

Freeman, D. M., Bhandarkar, V., Shinn, E., Wilkins-Wells, J., y Wilkins-Wells, P. (1989). Local Organizations for Social Development. Concepts and Cases of Irrigation Organization. Colorado: Westview Press. Recuperado de http:// pdf.usaid.gov/pdf_docs/PNABD173.pdf

Freeman, D. M., y Lowdermilk, M. L. (1985). Middle organizational linkage in irrigation projects. En M. Cernea (ed.), Putting People First (pp. 91-118). Washington: World Bank y Oxford University Press.

Gadea-Noguerón, R. (2019). La gestión social del agua para uso de riego en el río Yautepec (tesis de doctorado). Universidad Autónoma del Estado de Morelos. Recuperada de file:///C:/Users/jl/AppData/Local/Temp/GANRGN00T.pdf

Galindo-Escamilla, E., Bárcenas, R., y Palerm, J. (2018). ¿Una transferencia fallida? Respuestas organizativas de los usuarios para el manejo de las aguas negras del módulo Actopan. V Congreso de la Red-ISSA, El Colegio de San Luis, SLP.

Galindo-Escamilla, E., Bárcenas, R., y Palerm, J. (2019). Respuestas organizativas de los usuarios para el manejo de las aguas negras del Módulo de Riego Actopan. Memorias Las Ciencias Administrativas como Eje Estratégico en el Desarrollo de las Organizaciones. Universidad Michoacana de San Nicolás de Hidalgo.

Giménez Casalduero, M. , y Palerm, J. (2007). Organizaciones tradicionales de gestión del agua: importancia de su reconocimiento legal como motor de su pervivencia. región y sociedad, 19(38), 3-24. doi: 10.22198/rys.2007.38. a555 
Glick, T. (1972). Water for Larsa: an old Babylonian archive dealing with irrigation by Stanley D. Walters; the Negev: the challenge of a desert by Michael Evenari, Leslie Shananand Naphtali Tadmore. The Journal of Interdisciplinary History, 3(2), 416-420.

Grossi, P. (1981). An Alternative to Private Property: Collective Property in the Juridical Consciousness of the Nineteenth Century. Illinois: University of Chicago Press.

Gutiérrez Rivas, A. M., Romero-López, A. R., y Palerm, J. (eds.). (2017) [2004]. Catálogo de Reglamentos de Agua en México. Siglo XX. Recuperado de https://www.researchgate.net/publication/323004064_Catalogo_de_Reglamentos_de_Agua_en_Mexico_Siglo_XX_PRESENTACION

Hunt, R. C. (1997). Sistemas de riego por canales: tamaño del sistema y estructura de la autoridad. En T. Martínez Saldaña y J. Palerm (eds.), Antología sobre pequeño riego, Vol. 1 (pp. 185-219). México: Colegio de Postgraduados.

Lajaunie, M.-L., Tillier, S., Serrano Navarro, H. A., Lombana Córdoba, C., Scheierling, S., y Chinarro, L. (2013). El futuro del riego en Perú, Vol. II. Informe principal. Washington, DC: Serie de Publicaciones Ocasionales sobre Medio Ambiente y Recursos Hídricos y Banco Mundial.

Lanz Cárdenas, J. T. (ed.). (1982). Legislación de aguas en México. Estudio histórico-legislativo de 1521-1981, Vol. 1. México: Gobierno del Estado de Tabasco.

Mestries, F., y Bonilla, T. (2010). Crisis de sustentabilidad de la agricultura de riego en el valle central de Puebla. Estudios Agrarios, 16(43), 15-28.

Mikhail, A. (2019). An irrigated empire: the view from Ottoman Fayyum. Int. J. Middle East Stud., 42, 569-590. doi: 10.1017/S0020743810000826

Montes de Oca, A. (2020). Avances de investigación: Módulo Huapango, Distrito de Riego Arroyo Zarco. Manuscrito inédito.

Montes de Oca, A., y Palerm, J. (2013). Los reservorios secundarios (jagüeyes) en el sistema de riego Tepetitlán: el control local. En J. Palerm y T. Martínez Saldaña (eds.), Antología sobre riego: instituciones para la gestión del agua: vernáculas, alegales e informales (pp. 215-233). México: Biblioteca básica de agricultura, Colegio de Postgraduados y Grupo Mundi-Prensa.

Montes de Oca, A., Palerm, J., y Chávez-Mejía, M. C. (2010). Difficulties in the Tepetitlán irrigation system transfer, Estado de México (Las vicisitudes de la transferencia del sistema de riego Tepetitlán, Estado de México). Agricultura. Sociedad y Desarrollo, 4(2), 105-126.

Montes de Oca, A., Palerm, J., y Chávez-Mejía, M. C. (2012). El sistema de riego Tepetitlán, México: mejoras en la distribución del agua a partir de la transferencia. Tecnología y Ciencias del Agua, 3(1), 77-101.

Oré, T. (2005a). Agua, bien común y usos privados: riego, Estado y conflictos en la Achirana del Inca. Lima: Pontificia Universidad Católica del Perú, Wageningen University, Water Law and Indigenous Rights (WALIR) y Soluciones Práctica (ITDG).

Oré, T. (2005b). Estado, indígenas y hacendados ante el riego en un valle de la costa Peruana (1900-1930). Boletín Archivo Histórico del Agua, 10(29), 2743. 
Ostrom, E. (2011) [1990]. El gobierno de los bienes comunes. La evolución de las instituciones de acción colectiva. México: Fondo de Cultura Económica y SEMARNAP.

Palacios Vélez, E., Exebio García, A., Mejía Sáenz, E., Santos Hernández, A. L., y Delgadillo Piñón. (2002). Problemas financieros de las asociaciones de usuarios y su efecto en la conservación y operación de distritos de riego. Terra Latinoamericana, 20(4), 505-513.

Palerm, J. (1999). Detrás de los reglamentos formales: distribución del agua entre regantes autogestivos en situaciones de escasez. En J. Collado (ed.), Reglamentación de Sistemas de Riego, IX Congreso Nacional de Irrigación, Simposio 6 (pp. 73-79). México: ANEI.

Palerm, J. (2001). Organizational strategies in water shortage situations: Mexican self-administrated irrigation systems. International Journal of Water, 1(3-4).

Palerm, J. (2003). Organización autogestiva de regantes. En P. Ávila (ed.), Agua, ambiente y desarrollo en el siglo XXI, Vol. 2 (pp. 347-362). Zamora: El Colegio de Michoacán, IMTA y Secretaría de Urbanismo y Medio Ambiente.

Palerm, J. (2005). Gobierno y administración de sistemas de riego: tipologías. región y sociedad, 17(34), 3-33. doi: 10.22198/rys.2005.34.a614

Palerm, J. (2006). Self-management of irrigation systems, a typology: the Mexican case. Mexican Studies, 22(2), 361-385. doi: 10.1525/msem.2006.22.2.36

Palerm, J. (2009a). Distritos de riego en México, algunos mitos. En J. Palerm y T. Martínez Saldaña (eds.), Aventuras con el agua. La administración del agua de riego: historia y teoría (pp. 277-328). México: Colegio de Postgraduados.

Palerm, J. (2009b). Governance and management of irrigation systems. Water Policy, 11(3), 330-347.

Palerm, J. (2014). Are visible and strong legal frameworks always necessary to sustain irrigation institutions?: Some wider lessons for water resource management. WIREs Water, 1, 295-304. doi: 10.1002/wat2.1020

Palerm, J., Martínez-Saldaña, T., y Escobedo, J. F. (2000). Modelo de investigación: organización social de sistemas de riego en México. En J. Palerm-Viqueira y T. Martínez Saldaña (eds.), Antología sobre pequeño riego. Organizaciones autogestivas, Vol. 2 (pp. 31-62). México: Colegio de Postgraduados y Plaza y Valdés.

Palerm, J., Pimentel-Equihua, J. L., y Salcedo, I. (2000). Organización diferencial y escasez de agua: caso río Cuautla, Morelos. En J. Palerm-Viqueira y T. Martínez Saldaña (eds.), Antología sobre pequeño riego. Organizaciones autogestivas, Vol. 2 (pp. 73-96). México: Colegio de Postgraduados y Plaza y Valdés.

Palerm, J., Rodríguez-Haros, B., López Pacheco, E., Cháirez, C., Morán, L. del C., Osorio, R., Hernández-Rodríguez, M. de L., Sánchez-Almaraz, L. E., Pimentel-Equihua, J. L., Valdovinos-Ayala, J. H., y Sánchez, D. (2001). Informe técnico. Diagnóstico organizativo de la Asociación de Usuarios del río Cuautla (Morelos). Financiado por CNA, Núm. de convenio CNA-GDUR-CP-05/2001. 
Pedroza González, E., y Hinojosa Cuéllar, G. A. (2014). Manejo y distribución del agua en distritos de riego. Breve introducción didáctica. Jiutepec: Instituto Mexicano de Tecnología del Agua.

Pérez Magaña, A., Macías López, A., y Gutiérrez Villalpando, V. (2019). Situación social y tecnológica en el manejo del agua para riego en Puebla, México. Acta Universitaria, 29, 1-15. doi: 10.15174/au.2019.2114

Pimentel-Equihua, J. L. (2004). Los comuneros regantes de la acequia real del río Júcar en Valencia, España. Boletín Archivo Histórico del Agua: Organizaciones Autogestivas para el Riego. Nueva época, año 9, publicación de aniversario (10 años), 45-56.

Pimentel Equihua, J. L. (2020). Avances de investigación. Módulos del Distrito de Riego Zamora. Manuscrito inédito.

Roa García, M. C. (2014). Equity, efficiency and sustainability in water allocation in the Andes: trade-offs in a full world. Water Alternatives, 7(2), 298319.

Rodríguez Meza, J. G. (2000). El Comité de Vigilancia de los ríos Atoyac y Nexapa: formación de una organización. En J. Palerm-Viqueira y T. Martínez Saldaña (eds.), Antología sobre pequeño riego. Organizaciones autogestivas, Vol. 2 (pp. 345-406). México: Colegio de Postgraduados y Plaza y Valdés.

Romero Blake, A. A. (2008). Implicaciones sociales y culturales por la escasez de agua en la región del Papigochi, Chihuahua, en un contexto de sequía (tesis de maestría). ENAH, unidad Chihuahua y CIESAS. Recuperada de https://mediateca.inah.gob. $\mathrm{mx} /$ repositorio/islandora/object/tesis\%3A873

Romero-López, A. R., y Palerm, J. (2018). El rol de las organizaciones intermedias en la gestión del agua en México. V Congreso de la Red-ISSA, El Colegio de San Luis.

Salomón, E. (2020). Avances de investigación. Módulo Joachín y Distrito de Riego Río Blanco. Manuscrito inédito.

Sengupta, N. (2002). Institutions and engineering. Evolution of irrigation designs in some Asian countries. Conference on the environmental history of Asia. University of Sussex.

Torregrosa, Ma. L., y Salgado, A. (2018). Modernización y despojo en el Distrito de Riego de El Mayo Sonora. V Congreso de la Red-ISSA, El Colegio de San Luis, SLP.

Trava Manzanilla, J. L. (1995). La transferencia de los Distritos de Riego. Trabajo de ingreso a la Academia de Ingeniería de México. Recuperado de https://es.slideshare.net/AcademiaDelngenieriaMx/transferencia-de-los-distritos-de-riego

Trawick, P. (2001). The moral economy of water: equity and antiquity in the Andean commons. American Anthropologist, 103(2), 361-379.

Trawick, P. (2002). The moral economy of water: general principles for successfully managing the commons. GAIA, 11(3), 5-8.

Trawick, P. (2003). Against the privatization of water: an indigenous model for improving existing laws and successfully governing the commons. World Development, 31(6), 977-996. 
Vaidyanathan, A. (2009) [1985]. Instituciones de control del agua y agricultura: una perspectiva comparativa. En J. Palerm-Viqueira y T. Martínez Saldaña (eds.), Aventuras con el agua. La administración del agua de riego: historia y teoría (pp. 73-115). México: Colegio de Postgraduados.

Venado Godínez, M. G., y Palerm, J. (2013). Movilizaciones en época de estiaje en el Distrito de Riego 003 Tula. En J. Palerm y T. Martínez-Saldaña (eds.), Antología sobre riego: instituciones para la gestión del agua: vernáculas, alegales e informales (pp. 235-274). México: Biblioteca Básica de Agricultura, Colegio de Postgraduados y Grupo Mundi-Prensa.

Venado Godínez, M. G., Tavera Fenollosa, L., y Palerm, J. (2018). La participación de los regantes en la toma de decisiones sobre distribución de agua. $\mathrm{V}$ Congreso de la Red-ISSA, El Colegio de San Luis, SLP.

Villarreal, F., y Manzanal, M. (2011). Descentralización y poder en la administración del agua de riego. El caso de la cuenca del río Calchaquí en el departamento de San Carlos, Salta, Argentina. Campo Território: Revista de Geografia Agrária, 6(12), 32-62.

Wittfogel, K. (1966). Despotismo oriental. Estudio comparativo del poder totalitario. Madrid: Guadarrama.

Yoder, R. (1994a). Locally Managed Irrigation Systems. Sri Lanka: IIMI.

Yoder, R. (1994b). Organization and Management by Farmers in the Chhattis Mauja Irrigation System, Nepal. Sri Lanka: IIMI.

Zegarra, E. (2002). La investigación social sobre el manejo del agua de riego en el Perú: una mirada a conceptos y estudios empíricos. En M. Pulgar Vidal, E. Zegarra, y J. Urrutia (eds.), Perú, el problema agrario en debate: Seminario Permanente de Investigación Agraria (pp. 319-348). Lima: SEPIA.

Zegarra, E. (2004). Perú: viabilidad y potencialidad del mercado de agua. En A. Jouravlev (ed.), Mercados (de derechos) de agua: experiencias y propuestas en América del Sur (pp. 49-66). Santiago de Chile: Naciones Unidas.

Zenteno, G. (2020). Avances de investigación. Módulo y Distrito de Riego Cuxtepeques. Manuscrito inédito. 